\title{
Physical activity versus metformin for increasing insulin sensitivity in children and adolescents at-risk for type 2 diabetes
}

\author{
Andrew D Hanna, Natalie V Scime \\ Faculty Reviewer: Shauna Burke, PhD (Department of Health Studies)
}

\section{ABSTRACT}

Global rates of type 2 diabetes (T2D) among children and adolescents are steadily rising. As such, an increasing amount of attention and research has begun to focus on strategies to prevent this chronic and burdensome disease in pediatric populations. The purpose of this article is to briefly review current evidence pertaining to the effectiveness of physical activity versus metformin in improving insulin sensitivity of children at-risk (ie, obese and/or insulin resistant) for developing T2D. Potential barriers to each preventative intervention will also be discussed. Physical activity, both aerobic and resistance, has demonstrated effectiveness in a moderate number of demographically diverse pediatric studies. However, the pediatric population is already alarmingly sedentary with barriers such as lack of motivation, social stigma and discomfort presenting a challenge. A small number of studies have demonstrated the beneficial effects of metformin in children and adolescents for improved insulin sensitivity. However, longer and larger studies are required to confirm these findings and elucidate upon the long-term safety and efficacy of this pharmaceutical in pediatric populations. While no head-to-head studies examining physical activity and metformin exist in pediatric populations and more research is needed, current evidence seems to favour the use of physical activity given the larger quantity of studies and generalizability of its beneficial effects. Thus, physical activity should be emphasized in clinical and public health practice when targeting at-risk children and adolescents to prevent a T2D diagnosis.

\section{INTRODUCTION}

In the past two decades, global rates of type 2 diabetes (T2D) among children and adolescents have steadily risen. ${ }^{1}$ T2D leads to many acute and chronic health conditions, including increased risk of cardiovascular disease, hypertension, dyslipidemia, and microvascular complications. ${ }^{2}$ In Canada, the societal impact of diabetes is tremendous as it increases the demands of healthcare services, decreases productivity due to illness, and in many cases, causes premature death. ${ }^{3}$ The combined individual and economic burden of a T2D diagnosis underscore the importance of focusing efforts on reducing the incidence and prevalence of this chronic disease, particularly when it occurs early in life.

Several modifiable risk factors exist that may lead to an increased likelihood of T2D. Obesity represents a major risk factor and comorbidity in association with pediatric T2D, as in Canada, 95\% of children newly diagnosed with T2D are classified as obese. ${ }^{4}$ Further, insulin resistance, characterized as a decreased ability to appropriately respond to insulin, often occurs as a result of obesity and represents another risk factor for T2D. ${ }^{5}$ Prolonged insulin resistance, demonstrated by mildly elevated blood glucose levels that do not yet reach the threshold for a T2D diagnosis, can be characterized as prediabetes. ${ }^{6}$ Despite the progression from prediabetes to T2D occurring more quickly in children than in adults, studies have shown the potential to reverse insulin resistance and restore normal glucose metabolism if identified early.,7

Given the alterable nature of these factors, clinical practice guidelines from the Canadian Diabetes Association assert that early screening and intervention in children and adolescents at-risk for T2D (ie, obese and/or insulin resistant) is essential. ${ }^{2}$ When addressing T2D risk factors in adults, lifestyle modifications, such as physical activity and nutrition, and pharmacotherapy, such as the administration of metformin, are the two recommended interventions with cited efficacy. ${ }^{8}$ Unfortunately, the evidence examining these forms of preventive medicine in child and adolescent populations is sparse, and challenges exist with the individual uptake and adherence to both types of interventions. ${ }^{2,7}$ The purpose of this article is to briefly review current evidence pertaining to physical activity versus the use of metformin for improving insulin sensitivity in children that are at-risk for developing T2D. The barriers to implementation for physical activity interventions and metformin administration will also be briefly discussed.

\section{PHYSICAL ACTIVITY}

Lifestyle modifications in the form of physical activity have been recommended for adults diagnosed with T2D and at-risk for a diagnosis by the Canadian Diabetes Association. ${ }^{8}$ Benefits of physical activity include improved glycemic control, increased insulin sensitivity, improved lipid profile, blood pressure reduction, and improved maintenance of weight loss. ${ }^{9}$ While most evidence supports these benefits in adults, fewer studies have been conducted within the pediatric population. One meta-analysis of 24 randomized controlled trials containing over 1500 diabetic or insulin insensitive children (ages 6 to 19) showed a small to moderate effect when examining the effectiveness of exercise training on insulin resistance in adolescents. ${ }^{10}$ This beneficial effect remained consistent across age, gender, and race. Thorough analysis regarding the effect of frequency, duration, intensity, mode, or volume of exercise was not performed given the limited number of studies. Addressing this 
gap in knowledge, Kim and Park found in their review of the literature that despite inconclusive evidence, both resistance and aerobic exercise alone (without weight loss or calorie restriction) can effectively improve insulin sensitivity in children and adolescents at-risk for T2D. ${ }^{11}$ While the vast majority of studies on insulin resistance and physical activity have been conducted in adults, expert consensus suggests that these benefits are applicable to pediatric populations as well, further reinforcing the advantages of physical activity in reducing the incidence of $\mathrm{T} 2 \mathrm{D}$ in at-risk children and adolescents.

\section{CHALLENGES WITH PHYSICAL ACTIVITY IN PEDIATRIC POPULATIONS}

Despite the promising studies of physical activity interventions in addressing insulin resistance, several barriers exist that could limit population uptake. The pediatric population in Canada is already alarmingly sedentary, with only $9 \%$ of Canadian children and adolescents (ages 5 to 17 ) meeting physical activity guidelines ( $\geq$ 60 minutes of moderate-to-vigorous physical activity daily). ${ }^{12}$ One study examined adolescents (ages 10 to 17) who had been diagnosed with T2D two years prior concluded that the participants were significantly less physically active and spent more time being sedentary than similarly aged adolescents without diabetes. ${ }^{13}$ A recent review regarding perspectives on physical activity and exercise in diabetic adolescents concluded that lack of time and motivation, a mentality that the value of exercise does not outweigh the efforts of participating, social stigma associated with overweight adolescents, and body image factors are among several barriers..$^{14}$ Physical discomfort is also cited as a major barrier, and predominantly affects children with obesity. ${ }^{14}$ Interestingly, this discomfort has a physiologically substantiated basis, as impaired exercise kinetics among adolescents with T2D can make exercising at a beneficial intensity difficult and uncomfortable. ${ }^{15}$

\section{METFORMIN}

The administration of metformin is another viable avenue for reducing $\mathrm{T} 2 \mathrm{D}$ risk and improving insulin sensitivity in $\mathrm{T} 2 \mathrm{D}$ adult populations. ${ }^{16}$ Current literature regarding the safety and efficacy of metformin in pediatric populations is limited, and Canadian Diabetes Association clinical guidelines endorses the prescription of metformin after a diagnosis of T2D has been established and only if the desired glycemic targets have not been achieved after 3 to 6 months of lifestyle intervention. ${ }^{2}$ A meta-analysis of five studies examining the use of metformin in a total of 365 hyperinsulinemic (ie, excess levels of insulin circulating in the blood relative to glucose) or insulin resistant obese children and adolescents (ages 6 to 19) found a moderately beneficial effect on insulin resistance. ${ }^{17}$ Not surprisingly, the administration of metformin in pediatric patients is accompanied by the same plethora of side effects seen in adults, with $20-30 \%$ of participants in this pooled analysis reporting gastrointestinal problems. The authors of this meta-analysis emphasize the need to interpret these findings with caution, as included studies were short-term (ie, no longer than six months), based on small sample sizes largely comprised of ethnicities at a higher risk for T2D, and presented unadjusted measures with no intention to treat analysis. Echoing this sentiment, the Focus on a Fitter Future Committee, a subcommittee within the American Academy of Pediatrics, does not advocate for the use of pharmaceuticals to delay the onset of $\mathrm{T} 2 \mathrm{D}$ in pediatric populations with prediabetes due to the need for long-term, large scale, and demographically diverse studies. ${ }^{7}$

\section{CHALLENGES WITH METFORMIN USE IN PEDIATRIC POPULATIONS}

Challenges exist with prescribing metformin in pediatric populations at-risk for $\mathrm{T} 2 \mathrm{D}$, relating to the paucity of research and the general barriers to proper medication use and compliance. The use of pharmacotherapy in pediatric populations when only limited safety, efficacy, and dosage data exists, such as the case with metformin for children at-risk for T2D, may expose patients to an unnecessary risks and side effects. The World Health Organization asserts this notion in specifying that the pediatric population presents a different array of physiologies than that of adults, and the progressive developmental maturation and changes that occur during typical pediatric growth can influence the pharmacokinetics of various medications..$^{18}$ Further, evidence suggests that children and adolescents with chronic illness experience poor medication adherence, with the varying developmental capacities of this population being a strong influencer on adherence. ${ }^{19}$

\section{CONCLUSION}

The prevalence and incidence of childhood $\mathrm{T} 2 \mathrm{D}$ continues to rise in Canada. As such, efforts to reduce the severity and potential progression of $\mathrm{T} 2 \mathrm{D}$ in at-risk children and adolescents, such as those that are obese or insulin resistant, should be a health priority. Although studies related to the effectiveness of physical activity and metformin in the pediatric population are limited and more research is required, some important conclusions can be drawn. Physical activity in both aerobic and resistance forms represent a promising means to improve insulin sensitivity in pediatric populations, however, several barriers to consistent activity levels are pervasive in this already sedentary population. The few studies that examine metformin in pediatric populations have demonstrated its short-term effectiveness in improving insulin resistance in small-scale studies. Despite this, there is a need for long-term studies with diverse populations to safely establish the role of metformin as a preventive therapy in children and adolescents at-risk for T2D. Additionally, issues with prescribing metformin include some concerns about off-label use in certain pediatric patients and adherence challenges. It is important to note that no head-to-head studies could be located that examined the effectiveness of metformin versus physical activity interventions in preventing T2D among children and adolescents. Nonetheless, adult population studies have demonstrated that while combining lifestyle changes and metformin can reduce the incidence of T2D in persons at high risk, lifestyle intervention is more effective than metformin. ${ }^{20}$ 
Acknowledging this finding coupled with the current state of evidence, it is suggested that physical activity should be emphasized when attempting to prevent T2D in at-risk pediatric populations to effectively and safely preserve the health and livelihood of children.

\section{REFERENCES}

1. Nadeau K, Dabelea D. Epidemiology of Type 2 Diabetes in Children and Adolescents. Endocr Res. 2008;33(1-2):35-58.

2. Panagiotopoulos C, Riddell MC, Sellers EAC. Type 2 Diabetes in Children and Adolescents. Can J Diabetes. 2013;37:S163-7.

3. Public Health Agency of Canada. Diabetes in Canada: Facts and figures from a public health perspective; Chapter 3 - The health system and economic impact of diabetes [Internet]. Public Heal Agency Canada. 2011 [cited 2016 Mar 9]. Available from: http://www.phac-aspc.gc.ca/ cd-mc/publications/diabetes-diabete/facts-figures-faits-chiffres-2011/ chap3-eng.php

4. Public Health Agency of Canada. Diabetes in Canada: Facts and figures from a public health perspective; Chapter 5 - Diabetes in children and youth [Internet]. 2011 [cited 2016 Mar 9]. Available from: http://www. phac-aspc.gc.ca/cd-mc/publications/diabetes-diabete/facts-figuresfaits-chiffres-2011/chap5-eng.php

5. Chiarelli F, Marcovecchio ML. Insulin resistance and obesity in childhood. Eur J Endocrinol. 2008;159(SUPPL. 1):67-74.

6. Weiss R. Impaired glucose tolerance and risk factors for progression to type 2 diabetes in youth. Pediatr Diabetes. 2007 Dec;8 (Suppl 9):70-5.

7. Haemer M, Grow HM, Fernandez C, et al. Addressing prediabetes in childhood obesity treatment programs: support from research and current practice. Child Obes. 2014;10(4):292-303.

8. Ransom T, Goldenberg R, Mikalachki A, et al. Reducing the Risk of Developing Diabetes. Can J Diabetes. Canadian Diabetes Association; 2013;37:S16-9.

9. Sigal R, Armstrong MJ, Colby P, et al. Physical activity and diabetes. Can J Diabetes. 2013;37:S40-4.

10. Fedewa MV, Gist NH, Evans EM, et al. Exercise and Insulin Resistance in Youth: A Meta-Analysis. Pediatrics. 2014;133(1):E163-74.

11. Kim Y, Park H. Does regular exercise without weight loss reduce insulin resistance in children and adolescents? Int J Endocrinol. 2013;2013:402592.

12. Statistics Canada. Directly measured physical activity of children and youth, 2012 and 2013 [Internet]. Heal Fact Sheets. 2015 [cited 2016 Mar 26]. Available from: http://www.statcan.gc.ca/pub/82-625-x/2015001/ article/14136-eng.htm

13. Kriska A, Delahanty L, Edelstein S, et al. Sedentary Behavior and Physical Activity in Youth With Recent Onset of Type 2 Diabetes. Pediatrics. 2013;131(3):e850-6.

14. Pivovarov JA, Taplin CE, Riddell MC. Current perspectives on physical activity and exercise for youth with diabetes. Pediatr Diabetes. 2015;16(4):242-55.

15. Nadeau KJ, Zeitler PS, Bauer TA, et al. Insulin resistance in adolescents with type 2 diabetes is associated with impaired exercise capacity. J Clin Endocrinol Metab. 2009 Oct;94(10):3687-95.

16. Harper W, Clement M, Goldenberg R, et al. Pharmacologic Management of Type 2 Diabetes. Can J Diabetes. Canadian Diabetes Association; 2013;37:S61-8.

17. Park M, Kinra S, Ward K, et al. Metformin for Obesity in Children and Adolescents : A Systematic Review. Diabetes Care. 2009;32(9):1-3.

18. World Health Organization. Promoting Safety of Medicines for Children [Internet]. World Health. 2007 [cited 2016 Mar 9]. p. 59. Available from: http://www.who.int/medicines/publications/essentialmedicines/Promotion_safe_med_childrens.pdf

19. Dean AJ, Walters J, Hall A. A systematic review of interventions to enhance medication adherence in children and adolescents with chronic illness. Arch Dis Child. 2010;95(9):717-23.

20. Knowler WC, Barrett-Connor E, Fowler SE, et al. Reduction in the incidence of type 2 diabetes with lifestyle intervention or metformin. N Engl J Med. 2002;346(6):393-403. 